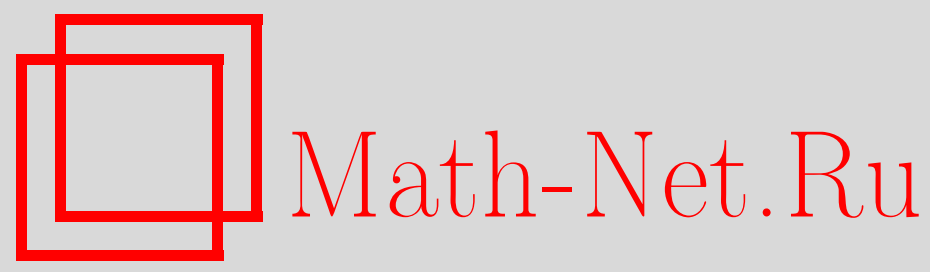

Е. А. Киреева, А. Н. Красильников, О некоторых экстремальных многообразиях ассоциативных алгебр, Матем. заметки, 2005, том 78, выпуск 4, 542-558

DOI: https://doi.org/10.4213/mzm2613

Использование Общероссийского математического портала Math-Net.Ru подразумевает, что вы прочитали и согласны с пользовательским соглашением http://www . mathnet.ru/rus/agreement

Параметры загрузки:

IP: 54.162 .127 .20

26 апреля 2023 г., 09:20:16

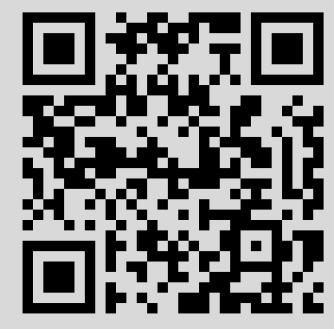




\section{О НЕКОТОРЫХ ЭКСТРЕМАЛЬНЫХ МНОГООБРАЗИЯХ АССОЦИАТИВНЫХ АЛГЕБР}

\section{Е. А. Киреева, А.Н. Красильников}

Пусть $F$ - поле простой характеристики $p, \mathbf{V}_{p}$ - многообразие ассоциативных алгебр над $F$, заданное тождествами $[[x, y], z]=0$ и $x^{p}=0$, если $p>2$, и тождествами $[[x, y], z]=0$ и $x^{4}=0$, если $p=2($ здесь $[x, y]=x y-y x)$. Известно, что свободные алгебры счетного ранга многообразия $\mathbf{V}_{p}$ содержат неконечнопорожденные $T$-пространства. Нами доказано, что многообразия $\mathbf{V}_{p}$ являются минимальными относительно указанного свойства.

Библиография: 18 названий.

1. Введение. Пусть $F$ - поле, $A$ - свободная ассоциативная $F$-алгебра без единицы счетного ранга со свободными порождающими $x_{1}, x_{2}, \ldots$ Элемент $v=v\left(x_{1}, \ldots\right.$, $\left.x_{n}\right) \in A$ называется полиномиальным тождеством или просто тождеством ассоциативной $F$-алгебры $G$, если $v\left(g_{1}, \ldots, g_{n}\right)=0$ для любых $g_{1}, \ldots, g_{n} \in G$. В этом случае выражение $v=0$ также назьвают тождеством алгебры $G$. Если $\left\{v_{t} \mid t \in \Omega\right\}-$ произвольное, но фиксированное множество тождеств, то класс всех ассоциативных $F$-алгебр, удовлетворяющих одновременно всем тождествам $v_{t}, t \in \Omega$, называется многообразием. Многообразие называется конечнобазируемым, если оно может быть определено конечным множеством тождеств, и неконечнобазируемым в противном случае.

Идеал $V$ свободной алгебры $A$ называется $T$-идеалом, если $V$ - вполне характеристический идеал в $A$, т.е. если $\alpha(V) \subseteq V$ для любого эндоморфизма $\alpha$ алгебры $A$. Хорошо известно, что между множеством всех многообразий ассоциативных $F$-алгебр и множеством всех $T$-идеалов алгебры $A$ существует естественное взаимнооднозначное соответствие. Именно, если $\mathbf{V}$ - многообразие ассоциативных $F$-алгебр, то соответствующий ему $T$-идеал $V$ алгебры $A$ - это множество всех тождеств, вьполненных в каждой алгебре из V. С другой стороны, если $V-T$-идеал в $A$, то соответствующее ему многообразие $\mathbf{V}$ - это многообразие, определяемое системой тождеств $\{v \mid v \in V\}$. Многообразие $\mathbf{V}$ ассоциативных $F$-алгебр является конечнобазируемым тогда и только тогда, когда соответствующий ему $T$-идеал $V$ конечнопорожден (как $T$-идеал). Основные определения, факты и библиографию, относящуюся к полиномиальньм тождествам и многообразиям ассоциативных алгебр, можно найти в [1]-[4].

В течение нескольких десятилетий оставалась открытой знаменитая проблема Шпехта (первоначально поставленная в [5] в случае, когда $F$ - поле характеристики 0$)$ : верно ли, что каждое многообразие ассоциативных $F$-алгебр конечнобазируемо? Или, эквивалентно: верно ли, что каждый $T$-идеал свободной $F$-алгебры $A$ счетного ранга конечнопорожден как $T$-идеал? Если $F$ - поле характеристики 0 , то ответ на этот вопрос 
положительньй, это было доказано в середине 80-х годов Кемером в замечательных работах [6] и [7]. С другой стороны, недавно Белов [8], Гришин [9] и Щиголев [10] показали, что над полем простой характеристики $p$ (над полем характеристики 2 в [9]) ответ на вопрос Шпехта отрищательньй (см. также [11], [12]). Ими были построены примеры $T$-идеалов в алгебре $A$, не являющихся конечнопорожденньми.

Пусть $\mathbf{V}$ - многообразие ассоциативных $F$-алгебр, $V$ - соответствующий этому многообразию $T$-идеал в $A$. Факторалгебра $A / V$ с порождающими $x_{1}+V, x_{2}+V, \ldots$ назьвается свободной алгеброй (счетного ранга) многообразия V. Векторное подпространство $U$ в $A / V$ назьвается $T$-пространством, если $U$ - вполне характеристическоеподпространство, т.е. $\alpha(U) \subseteq U$ для любого эндоморфизма $\alpha$ алгебры $A / V$. Первым систематически изучать $T$-пространства, прежде всего с точки зрения их конечной порожденности, начал Гришин [13].

Между проблемой Шпехта и вопросом о сушествовании неконечнопорожденных $T$ пространств в свободных алгебрах многообразий существует тесная связь. В каждой из работ [8], [9], [10] было построено свое неконечнобазируемое многообразие $\mathbf{U}$, отличное от построенньх в двух других работах, однако в каждой из этих работ при построении такого неконечнобазируемого многообразия $\mathbf{U}$ решающую роль играло вспомогательное многообразие $\mathbf{V}$, свободная алгебра $A / V$ счетного ранга которого содержит неконечнопорожденные $T$-пространства.

Минимальные неконечнобазируемые многообразия - это неконечнобазируемые многообразия, все собственные подмногообразия которых конечнобазируемы. Такие минимальные многообразия называются также почти конечнобазируемы.ми или предельными. С помощью леммы Цорна легко доказать, что каждое неконечнобазируемое многообразие универсальных алгебр содержит минимальное неконечнобазируемое подмногообразие. Поэтому из отрицательного решения проблемы Шпехта над полем $F$ простой характеристики вытекает, что указанные минимальные многообразия ассоциативньх $F$-алгебр существуют. Ни одного конкретного примера такого многообразия, однако, неизвестно. Поэтому сейчас особую актуальность приобрела следующая (по-видимому, очень трудная) проблема.

ПроБлЕмА 1. Построить минимальное неконечнобазируемое многообразие ассоциативных алгебр над полем $F$ простой характеристики.

Так как отправной точкой в построении неконечнобазируемого многообразия $\mathbf{U}$ acсоциативных алгебр надполем простой характеристики является вспомогательноемногообразие $\mathbf{V}$, свободная алгебра (счетного ранга) $A / V$ которого содержит неконечнопорожденные $T$-пространства, то для построения минимального неконечнобазируемого многообразия $\mathbf{U}$ в качестве первого шага нужно решить следующую проблему.

ПроБлЕма 2. Построить минимальное многообразие $\mathbf{V}$ ассоциативных алгебр над полем $F$ простой характеристики, свободная алгебра счетного ранга $A / V$ которого содержит неконечнопорожденные $T$-пространства.

Другими словами, требуется построить такое многообразие $\mathbf{V}$, что в свободной алгебре счетного ранга $A / V$ этого многообразия неконечнопорожденные $T$-пространства есть, а в свободной алгебре счетного ранга всякого собственного подмногообразия многообразия $\mathbf{V}$ все $T$-пространства конечнопорождены. Решение этой второй проблемы и составляет основной результат данной работы. 
Пусть $F$ - поле простой характеристики $p$; обозначим символом $\mathbf{V}_{p}$ многообразие ассоциативньх алгебр над $F$, заданное тождествами

$$
[[x, y], z]=0 \quad \text { и } \quad x^{p}=0
$$

если $p>2$, и тождествами

$$
[[x, y], z]=0 \quad \text { и } \quad x^{4}=0,
$$

если $p=2$ (здесь $[x, y]=x y-y x)$. Отметим, что для $p>2$ многообразие $\mathbf{V}_{p}$ порождается бесконечномерной алгеброй Грассмана над полем $F$ (Чирипов, Сидеров [14]).

Известно, что свободная алгебра счетного ранга многообразия $\mathbf{V}_{p}$ содержит неконечнопорожденные $T$-пространства (Гришин [9] для $p=2$ и Щиголев [15] для простого $p>2)$. Нами доказана

Теорема 1. Пусть $F$ - произвольное поле простой характеристики р. Многообразие $\mathbf{V}_{p}$ является минимальным многообразием ассоциативных $F$-алгебр, свободная алгебра счетного ранга которого содержит неконечнопорожсденные Т-пространства.

Утверждение теоремы 1 вытекает из следующей теоремы.

ТЕОРема 2. Пусть W - произвольное собственное подмногообразие многообразия $\mathbf{V}_{p}$. Тогда в свободной алгебре многообразия $\mathbf{W}$ любое T-пространство конечнопорохдено.

В свою очередь, теорема 2 является непосредственным следствием следующих двух утверждений, представляющих независимьй интерес.

ТЕОремА 3. Любое собственное подмногообразие $\mathbf{W}$ многообразия $\mathbf{V}_{p}$ удовлетворяет тохдеству

$$
\left[x_{1}, x_{2}\right] \cdot\left[x_{3}, x_{4}\right] \cdots\left[x_{2 n-1}, x_{2 n}\right]=0
$$

для подходящего $n=n(\mathbf{W})$.

Tеорема 4. Пусть $n, N \in \mathbb{N}, u$ nусть $\mathbf{V}=\mathbf{V}(n, N)$ - многообразие ассоииативных F-алгебр, определяемое тожсдствами (1) $и x^{N}=0$. Тогда в свободных алгебрах многообразия $\mathbf{V}$ все T-пространства конечнопорохсдены.

Заметим, что основные определения, связанные с многообразиями ассоциативных алгебр над полем, без изменений переносятся на алгебры над произвольным коммутативно-ассоциативным кольцом $K$ с 1 . Пусть $A / V$ - свободная алгебра многообразия $\mathbf{V}$ ассоциативных $K$-алгебр, свободно порожденная элементами $x_{i}+V, i \in \Omega$. Назовем $T$-пространство в $A / V$ ограниченным (относительно множества порождающих $\left.\left\{x_{i}+V \mid i \in \Omega\right\}\right)$, если оно порождается как $T$-пространство элементами, в которые каждьй порождающий $x_{i}+V$ входит в степени, не превышающей некоторого числа $N$, не зависящего от $i$.

Утверждение теоремы 4 вытекает из следующего результата, доказанного первым из авторов. 
Теорема 5. Пусть $K$ - произвольное нётерово коммутативное и ассоциативное кольио с $1, n$ - произвольное, но фиксированное натуральное число. Пусть $\mathbf{V}=\mathbf{V}(n)$ - многообразие ассочиативных алгебр над $K$, задаваемое тождеством (1). Тогда в свободных алгебрах многообразия $\mathbf{V}$ все ограниченные $T$-пространства конечнопорождены (как T-пространства).

2. Доказательство теоремы 3. Пусть $A / V_{p}$ - свободная алгебра счетного ранга многообразия $\mathbf{V}_{p}$. Пусть $T$-произвольный нетривиальньй $T$-идеал в $A / V_{p}$. Нам нужно доказать, что $T$ содержит элемент

$$
\left[x_{1}, x_{2}\right] \cdot\left[x_{3}, x_{4}\right] \cdots\left[x_{2 n-1}, x_{2 n}\right]+V_{p}
$$

для подходящего $n=n(T)$.

Рассмотрим в $A / V_{p}$ подмножество $H$ всех элементов

$$
x_{i_{1}}^{\alpha_{1}} \cdots x_{i_{l}}^{\alpha_{l}}\left[x_{j_{1}}, x_{j_{2}}\right] \cdots\left[x_{j_{2 m-1}}, x_{j_{2 m}}\right]+V_{p}
$$

таких, что $l, m \in \mathbb{Z}, l, m \geqslant 0, l+m>0, i_{1}<\cdots<i_{l}, j_{1}<\cdots<j_{2 m}, \alpha_{t} \in\{1,2, \ldots, p-1\}$, если $p>2$, и $\alpha_{t} \in\{1,2,3\}$, если $p=2(t=1,2, \ldots, l)$. Покажем, что алгебра $A / V_{p}$ является линейной оболочкой подмножества $H$.

Действительно, рассмотрим произвольный элемент $f$ алгебры $A / V_{p}$. Этот элемент является линейной комбинацией одночленов вида $x_{u_{1}} \cdots x_{u_{s}}+V_{p}, s>0$. Используя равенства

$$
x y=y x+[x, y] \quad \text { и } \quad[x, y] z+V_{p}=z[x, y]+V_{p},
$$

каждый такой одночлен нетрудно записать в виде суммы элементов вида

$$
x_{i_{1}}^{\alpha_{1}} \cdots x_{i_{l}}^{\alpha_{l}}\left[x_{j_{1}}, x_{j_{2}}\right] \cdots\left[x_{j_{2 m-1}}, x_{j_{2 m}}\right]+V_{p},
$$

где $i_{1}<\cdots<i_{l}, m \in \mathbb{Z}, l, m \geqslant 0, l+m>0$. Ясно, что $1 \leqslant \alpha_{i}<4$, если $p=2$, и $1 \leqslant \alpha_{i}<p$, если $p>2$, иначе произведение равно 0 .

Хорошо известно (см. $[16])$, что в алгебре с тождеством $[[x, y], z]=0$ имеют место тождества

$$
\begin{aligned}
{[x, z] \cdot[y, t] } & =-[x, t] \cdot[y, z], \\
{[x, y] \cdot[x, z] } & =0 .
\end{aligned}
$$

Действительно,

$$
\begin{aligned}
0 & =[x y, z, t]=[x[y, z]+[x, z] y, t]=[x[y, z], t]+[[x, z] y, t] \\
& =x[y, z, t]+[x, t] \cdot[y, z]+[x, z] \cdot[y, t]+[x, z, t] y=[x, t] \cdot[y, z]+[x, z] \cdot[y, t] .
\end{aligned}
$$

Тождество (4) доказано. Положим в нем теперь $t=x$, тогда

$$
[x, z] \cdot[y, x]=-[x, x] \cdot[y, z]=0,
$$

откуда получаем тождество (5). 
Из тождества (5) следует, что произведения вида (3), в которых индексы $j_{1}, \ldots, j_{2 m}$ не являются попарно различньми, равны 0 . Если в произведении $(3)$ индексы $j_{1}, \ldots, j_{2 m}$ попарно различны, то используя тождество (4), порождающие $x_{j_{1}}, \ldots, x_{j_{2 m}}$ в этих произведениях можно упорядочить так, что $j_{1}<j_{2}<\cdots<j_{2 m}$. Таким образом, множество $H$ действительно порождает $A / V_{p}$ как векторное пространство над полем $F$. Известно (хотя мы не смогли найти точной ссылки), что элементы из множества $H$ линейно независимы над полем $F$. Мы, однако, не будем использовать этот факт. Наоборот, из нашего доказательства (с учетом того, что $\mathbf{V}_{p}$ не удовлетворяет никакому тождеству вида (1)) будет следовать, что любая нетривиальная линейная комбинация элементов $H$ ненулевая, и потому элементы из множества $H$ линейно независимы и составляют $F$-базис алгебры $A / V_{p}$.

Пусть $p=2$. Тогда для любых $u, v \in A / V_{2}$ имеем $\left[u, v^{2}\right]=[[u, v], v]=0$, т.е. $v^{2}$ лежит в центре алгебры $A / V_{2}$. Значит, любое произведение $g$ из множества $H$ можно переписать в виде

$$
g=g_{1} g_{2} g_{3},
$$

где

$$
\begin{gathered}
g_{1}=x_{i_{1}} \cdots x_{i_{l}}+V_{2}, \quad i_{1}<\cdots<i_{l}, \quad g_{2}=x_{j_{1}}^{2} \cdots x_{j_{m}}^{2}+V_{2}, \quad j_{1}<\cdots<j_{m} \\
g_{3}=\left[x_{k_{1}}, x_{k_{2}}\right] \cdots\left[x_{k_{2 r-1}}, x_{k_{2 r}}\right]+V_{2}, \quad k_{1}<\cdots<k_{2 r}
\end{gathered}
$$

при этом некоторые (но не все) множители $g_{i}$ могут отсутствовать. Назовем множители $g_{1}, g_{2}$ и $g_{3}$ соответственно начальной, средней и конечной частью произведения $g$. Число $l$ назовем длиной произведения $g_{1}$, а число $m$ - длиной произведения $g_{2}$.

Будем говорить, что порождающие $x_{i_{1}}+V_{2}, \ldots, x_{i_{l}}+V_{2}$ входят в указанноевышепроизведение $g_{1}$, а все остальные порождающие $x_{j}+V_{2}$ (т.е. порождающие с $j \notin\left\{i_{1}, \ldots, i_{l}\right\}$ ) не входят в это произведение. Аналогично, будем говорить, что в $g_{2}$ входят порождающие $x_{j_{1}}+V_{2}, \ldots, x_{j_{m}}+V_{2}$ и только они, а в $g_{3}$ - порождаюшие $x_{k_{1}}+V_{2}, \ldots, x_{k_{2 r}}+V_{2}$ и только они.

Рассмотрим эндоморфизмы $\varphi=\varphi_{i, s, t}: A / V_{2} \rightarrow A / V_{2}$ такие, что

$$
\varphi\left(x_{i}+V_{2}\right)=x_{i}+\left[x_{s}, x_{t}\right]+V_{2}, \quad \varphi\left(x_{j}+V_{2}\right)=x_{j}+V_{2} \quad \text { для всех } j \neq i .
$$

Нетрудно проверить, что если $g$ - произведение вида (6), то

$$
\varphi(g)= \begin{cases}g, & \text { если } x_{i}+V_{2} \text { не входит в } g_{1}, \\ g+\tilde{g}_{1} g_{2} g_{3}\left[x_{s}, x_{t}\right], & \text { если } x_{i}+V_{2} \text { входит в } g_{1} .\end{cases}
$$

Здесь $\tilde{g}_{1}$ - произведение, полученное из $g_{1}$ отбрасьванием множителя $x_{i}$. Получаем, что

$$
g+\varphi(g)= \begin{cases}0, & \text { если } x_{i}+V_{2} \text { не входит в } g_{1}, \\ \tilde{g}_{1} g_{2} g_{3}\left[x_{s}, x_{t}\right], & \text { если } x_{i}+V_{2} \text { входит в } g_{1} .\end{cases}
$$

Рассмотрим эндоморфизмы $\psi=\psi_{i, s}: A / V_{2} \rightarrow A / V_{2}$ такие, что

$$
\psi\left(x_{i}+V_{2}\right)=x_{i}+x_{s}+V_{2}, \quad \psi\left(x_{j}+V_{2}\right)=x_{j}+V_{2} \quad \text { для всех } j \neq i .
$$


Пусть $g$ - произведение вида (6), у которого отсутствует начальная часть, т.е.

$$
g=g_{2} g_{3}
$$

Нетрудно проверить, что тогда

$$
\psi(g)= \begin{cases}g, & \text { если } x_{i}+V_{2} \text { не входит в } g_{2}, g_{3}, \\ \left(x_{i}^{2}+x_{s}^{2}+\left[x_{i}, x_{s}\right]\right) \tilde{g}_{2} g_{3}, & \text { если } x_{i}+V_{2} \text { входит только в } g_{2}, \\ \left(\left[x_{i}, x_{j}\right]+\left[x_{s}, x_{j}\right]\right) g_{2} \tilde{g}_{3}, & \text { если } x_{i}+V_{2} \text { входит только в } g_{3}, \\ \left(x_{i}^{2}+x_{s}^{2}\right)\left(\left[x_{i}, x_{j}\right]+\left[x_{s}, x_{j}\right]\right) \tilde{g}_{2} \tilde{g}_{3}, & \text { если } x_{i}+V_{2} \text { входит и в } g_{2}, \text { и в } g_{3} .\end{cases}
$$

Здесь $\tilde{g}_{2}$ - произведение, полученное из $g_{2}$ отбрасьванием множителя $x_{i}^{2}$, а $\tilde{g}_{3}$ - произведение, полученное из $g_{3}$ отбрасьванием множителя $\left[x_{i}, x_{j}\right]$.

Рассмотрим также эндоморфизмы $\chi=\chi_{i, s}: A / V_{2} \rightarrow A / V_{2}$ такие, что

$$
\chi\left(x_{i}+V_{2}\right)=x_{s}+V_{2}, \quad \chi\left(x_{j}+V_{2}\right)=x_{j}+V_{2} \quad \text { для всех } j \neq i .
$$

Ясно, что

$$
g+\psi(g)+\chi(g)= \begin{cases}g, & \text { если } x_{i}+V_{2} \text { не входит в } g_{2}, g_{3}, \\ \tilde{g}_{2} g_{3}\left[x_{i}, x_{s}\right], & \text { если } x_{i}+V_{2} \text { входит только в } g_{2}, \\ 0, & \text { если } x_{i}+V_{2} \text { входит только в } g_{3}, \\ \left(x_{i}^{2}\left[x_{s}, x_{j}\right]+x_{s}^{2}\left[x_{i}, x_{j}\right]\right) \tilde{g}_{2} \tilde{g}_{3}, & \text { если } x_{i}+V_{2} \text { входит и в } g_{2}, \text { и в } g_{3} .\end{cases}
$$

Далее, нетрудно проверить, что если $g$ - произведение вида (7), $x_{s}+V_{2}, x_{t}+V_{2}-$ порождающие $A / V_{2}$, не входящие в $g$,

$$
f_{1}=g+\psi_{i, s}(g)+\chi_{i, s}(g), \quad f_{2}=f_{1}+\psi_{s, t}\left(f_{1}\right)+\chi_{s, t}\left(f_{1}\right),
$$

то

$$
f_{2}= \begin{cases}g, & \text { если } x_{i}+V_{2} \text { не входит в } g_{2}, g_{3}, \\ 0, & \text { если } x_{i}+V_{2} \text { входит только в } g_{2} \text { или только в } g_{3}, \\ \tilde{g}_{2} g_{3}\left[x_{s}, x_{t}\right], & \text { если } x_{i}+V_{2} \text { входит и в } g_{2}, \text { и в } g_{3} .\end{cases}
$$

Пусть теперь $T$ - произвольньй ненулевой $T$-идеал в алгебре $A / V_{2}$. Пусть $f=$ $\sum_{t} \alpha_{t} g^{(t)} \in T$, где для всех $t \alpha_{t} \in F, \alpha_{t} \neq 0, g^{(t)}$ - попарно различные произведения вида (6). Покажем, что без ограничения общности можно считать, что для некоторого $q \in \mathbb{N}$ в каждое из произведений $g^{(t)}$ входит каждый порождающий $x_{1}+V_{2}, \ldots, x_{q}+V_{2}$ и не входят никакие порождаюшие $x_{j}+V_{2}$ для $j>q$.

Действительно, пусть $g^{(1)}=g^{(1)}\left(x_{j_{1}}, \ldots, x_{j_{q}}\right)+V_{2}$, и пусть $\tau=\tau_{j_{1}, \ldots, j_{q}}$ - эндоморфизм алгебры $A / V_{2}$ такой, что

$$
\tau\left(x_{j_{t}}+V_{2}\right)=x_{j_{t}}+V_{2} \text { для } t=1,2, \ldots, q, \quad \tau\left(x_{j}+V_{2}\right)=0 \text { для } j \notin\left\{j_{1}, \ldots, j_{q}\right\} .
$$

Заменяя, если необходимо, $f$ на $\tau(f) \in T$, мы можем считать, что для любого $i$ в $g^{(i)}$ не входят никакие порождающие $x_{j}+V_{2}$ для $j \notin\left\{j_{1}, \ldots, j_{q}\right\}$. 
Далее, пусть $\xi_{i}$ - эндоморфизмы алгебры $A / V_{2}$ такие, что

$$
\xi_{i}\left(x_{j}+V_{2}\right)=x_{j}+V_{2} \text { для } j \neq i, \quad \xi_{i}\left(x_{i}+V_{2}\right)=0 .
$$

Легкопроверить, что $f_{1}=f+\xi_{j_{1}}(f) \in T-$ сумма тех слагаемых $\alpha_{t} g^{(t)}$ из $f$, для которых порождающий $x_{j_{1}}+V_{2}$ входит в $g^{(t)}$. Рассматривая далее $f_{2}=f_{1}+\xi_{j_{2}}\left(f_{1}\right) \in T$ и так далее, мы в результате получим элемент $f_{q}=\sum_{i} \alpha_{i} g^{(i)} \in T$, где $g^{(i)}-$ попарно различные произведения вида (6), в каждое из которых входят в точности порождающие $x_{j_{1}}+V_{2}, \ldots, x_{j_{q}}+V_{2}$ и не входят никакие другие.

И наконец, мы можем без ограничения общности считать $j_{1}=1, \ldots, j_{q}=q$, так как если $j_{k} \neq k$ для некоторого $k$, то достаточно заменить имеющийся элемент $f$ на $\sigma(f) \in T$ для подходящего автоморфизма $\sigma$ алгебры $A / V_{2}$, действующего как подстановка на множестве $\left\{x_{j}+V_{2} \mid j \in \mathbb{N}\right\}$.

Пусть теперь $f=\sum_{t=1}^{s} \alpha_{t} g^{(t)} \in T$, где для всех $t=1, \ldots, s \alpha_{t} \in F, \alpha_{t} \neq 0$, $g^{(t)}=g^{(t)}\left(x_{1}, \ldots, x_{q}\right)+V_{2}$ - попарно различные произведения вида $(6)$. Положим

$$
M_{0}=\left\{g^{(t)} \mid t=1, \ldots, s\right\} .
$$

Для всех $t$ обозначим символом $l_{t}$ длину начальной части $g_{1}^{(t)}$ произведения $g^{(t)}$, символом $m_{t}$ - длину средней части $g_{2}^{(t)}$ произведения $g^{(t)}$, символом $D^{(t)}$ - множество всех таких порождающих $x_{i}+V_{2}$ алгебры $A / V_{2}$, которые входят одновременно в среднюю и конечную части произведения $g^{(t)}$. Пусть $r_{t}=\left|D^{(t)}\right|$.

Пусть $\max _{t=1, \ldots, s} l_{t}=l_{\max }$. Положим

$$
M_{1}=\left\{g^{(t)} \in M_{0} \mid l_{t}=l_{\max }\right\} .
$$

Будем считать, что $g^{(t)} \in M_{1}$ при $t=1, \ldots, s_{1}, s_{1} \leqslant s$.

Пусть $\max _{t=1, \ldots, s_{1}} r_{t}=r_{\max }$. Положим

$$
M_{2}=\left\{g^{(t)} \in M_{1} \mid r_{t}=r_{\max }\right\} .
$$

Будем считать, что $g^{(t)} \in M_{2}$ при $t=1, \ldots, s_{2}, s_{2} \leqslant s_{1}$.

И, наконец, пусть $\max _{t=1, \ldots, s_{2}} m_{t}=m_{\max }$. Положим

$$
M_{3}=\left\{g^{(t)} \in M_{2} \mid m_{t}=m_{\max }\right\} .
$$

Зафиксируем теперь элемент $g^{(1)} \in M_{3}$. Пусть $g_{1}^{(1)}=x_{i_{1}} \cdots x_{i_{l}}+V_{2}, l=l_{\max }$. Тогда положим

$$
f_{0}^{(1)}=f, \quad f_{1}^{(1)}=f_{0}^{(1)}+\varphi_{i_{1}, q+1, q+2}\left(f_{0}^{(1)}\right) .
$$

Ясно, что $f_{1}^{(1)} \in T$, причем $f_{1}^{(1)}=\sum_{t} \alpha_{t}\left(g^{(t)}+\varphi_{i_{1}, q+1, q+2}\left(g^{(t)}\right)\right)$ и

$$
g^{(t)}+\varphi_{i_{1}, q+1, q+2}\left(g^{(t)}\right)= \begin{cases}0, & \text { если } x_{i_{1}}+V_{2} \text { не входит в } g_{1}^{(t)} \\ \tilde{g}_{1}^{(t)} g_{2}^{(t)} g_{3}^{(t)}\left[x_{q+1}, x_{q+2}\right], & \text { если } x_{i_{1}}+V_{2} \text { входит в } g_{1}^{(t)} .\end{cases}
$$

Положим далее

$$
f_{2}^{(1)}=f_{1}^{(1)}+\varphi_{i_{2}, q+3, q+4}\left(f_{1}^{(1)}\right), \quad \ldots, \quad f_{l}^{(1)}=f_{l-1}^{(1)}+\varphi_{i_{l}, q+2 l-1, q+2 l}\left(f_{l-1}^{(1)}\right) .
$$


Рассмотрим элемент $f_{l}^{(1)} \in T$. Так как для всех $t=s_{1}+1, \ldots, s$ выполняется $l_{t}<l$, то для каждого произведения $g^{(t)} \in M_{0} \backslash M_{1}$ найдется порождаюший $x_{i_{j}}+V_{2}$, который входит в $g_{1}^{(1)}$, но не входит в $g_{1}^{(t)}$, и, таким образом, все элементы из $M_{0} \backslash M_{1}$ перейдут в 0 .

Рассмотрим теперь элементы из $M_{1}$. Ясно, что для всякого $t=1, \ldots, s_{1}$ либо $g_{1}^{(t)} \neq g_{1}^{(1)}$, и тогда произведение $g_{1}^{(t)}$ также перейдет в 0 , либо $g_{1}^{(t)}=g_{1}^{(1)}$, и тогда вместо $g^{(t)}$ появится произведение $\bar{g}^{(t)}=g_{2}^{(t)} \bar{g}_{3}^{(t)}$, где

$$
\bar{g}_{3}^{(t)}=g_{3}^{(t)}\left[x_{q+1}, x_{q+2}\right] \cdots\left[x_{q+2 l-1}, x_{q+2 l}\right] .
$$

Применив, если нужно, подходяшие эндоморфизмы $\tau$ и $\xi$ к элементу $f_{l}^{(1)}$, отбросим все такие произведения $\bar{g}^{(t)}$, у которых набор входящих в них порождающих не совпадает с набором порождающих, входящих в $\bar{g}^{(1)}$. Применим также подходящий автоморфизм $\sigma$ и перенумеруем порождающие. Тогда, перенумеровывая, если необходимо, элементы $\alpha_{t} g^{(t)}$ в $f$, получаем

$$
\bar{f}=\sum_{t=1}^{s^{\prime}} \alpha_{t} h^{(t)} \in T, \quad \text { где } h^{(t)}=\sigma\left(\bar{g}^{(t)}\right),
$$

$h^{(t)}=h_{2}^{(t)} h_{3}^{(t)}=h^{(t)}\left(x_{1}, \ldots, x_{q_{1}}\right)+V_{2}$ - элементы вида $(7)$.

Положим

$$
\begin{aligned}
& \bar{M}_{1}=\left\{h^{(t)} \mid t=1, \ldots, s^{\prime}\right\}, \\
& \bar{M}_{2}=\left\{h^{(t)} \in \bar{M}_{1} \mid g^{(t)} \in M_{2}\right\}, \\
& \bar{M}_{3}=\left\{h^{(t)} \in \bar{M}_{2} \mid g^{(t)} \in M_{3}\right\} .
\end{aligned}
$$

Ясно, что $\bar{M}_{3} \neq \varnothing$, так как $h^{(1)} \in \bar{M}_{3}$.

Пусть $r=r_{\max }$, и пусть $x_{j_{1}}+V_{2}, \ldots, x_{j_{r}}+V_{2}$ - элементы из $D^{(1)}$, т.е. порождающие алгебры $A / V_{2}$, которые одновременно входят в $h_{2}^{(1)}$ и $h_{3}^{(1)}$.

Положим

$$
\begin{aligned}
f_{0}^{(2)} & =\bar{f} \\
f_{1}^{(2)} & =f_{0}^{(2)}+\psi_{j_{1}, q_{1}+1}\left(f_{0}^{(2)}\right)+\chi_{j_{1}, q_{1}+1}\left(f_{0}^{(2)}\right) \\
& =\sum_{t} \alpha_{t}\left(h^{(t)}+\psi_{j_{1}, q_{1}+1}\left(h^{(t)}\right)+\chi_{j_{1}, q_{1}+1}\left(h^{(t)}\right)\right)=\sum_{t} \alpha_{t} \bar{h}^{(t)}, \\
f_{2}^{(2)} & =f_{1}^{(2)}+\psi_{q_{1}+1, q_{1}+2}\left(f_{1}^{(2)}\right)+\chi_{q_{1}+1, q_{1}+2}\left(f_{1}^{(2)}\right) \\
& =\sum_{t} \alpha_{t}\left(\bar{h}^{(t)}+\psi_{q_{1}+1, q_{1}+2}\left(\bar{h}^{(t)}\right)+\chi_{q_{1}+1, q_{1}+2}\left(\bar{h}^{(t)}\right)\right)=\sum_{t} \alpha_{t} \overline{\bar{h}}^{(t)} .
\end{aligned}
$$

Тогда

$$
\overline{\bar{h}}^{(t)}= \begin{cases}0, & \text { если } x_{j_{1}}+V_{2} \text { входит только в } h_{2}^{(t)} \\ \tilde{h}_{2}^{(t)} h_{3}^{(t)}\left[x_{q_{1}+1}, x_{q_{1}+2}\right], & \text { если только в } h_{3}^{(t)},\end{cases}
$$


Положим далее

$$
\begin{aligned}
& f_{3}^{(2)}=f_{2}^{(2)}+\psi_{j_{2}, q_{1}+3}\left(f_{2}^{(2)}\right)+\chi_{j_{2}, q_{1}+3}\left(f_{2}^{(2)}\right) \\
& f_{4}^{(2)}=f_{3}^{(2)}+\psi_{q_{1}+3, q_{1}+4}\left(f_{3}^{(2)}\right)+\chi_{q_{1}+3, q_{1}+4}\left(f_{3}^{(2)}\right) \\
& \ldots \ldots \ldots \ldots \ldots \ldots \ldots \ldots \ldots \ldots \ldots \ldots \ldots \ldots \ldots \ldots \ldots \ldots \ldots \ldots \ldots \ldots \ldots \ldots \ldots \ldots \ldots \ldots \ldots \ldots \ldots \\
& f_{2 r-1}^{(2)}=f_{2 r-2}^{(2)}+\psi_{j_{r}, q_{1}+2 r-1}\left(f_{2 r-2}^{(2)}\right)+\chi_{j_{r}, q_{1}+2 r-1}\left(f_{2 r-2}^{(2)}\right), \\
& f_{2 r}^{(2)}=f_{2 r-1}^{(2)}+\psi_{q_{1}+2 r-1, q_{1}+2 r}\left(f_{2 r-1}^{(2)}\right)+\chi_{q_{1}+2 r-1, q_{1}+2 r}\left(f_{2 r-1}^{(2)}\right) .
\end{aligned}
$$

Ясно, что $f_{2 r}^{(2)} \in T$. Так как для всех $h^{(t)} \in \bar{M}_{1} \backslash \bar{M}_{2}$ выполняется $r_{t}<r$, то найдется порождающий $x_{j_{k}}+V_{2} \in D^{(1)}$ такой, что $x_{j_{k}}+V_{2} \notin D^{(t)}$ (т.е. $x_{j_{k}}+V_{2}$ входит либо только в $h_{2}^{(t)}$, либо только в $\left.h_{3}^{(t)}\right)$, следовательно, все элементы из $\bar{M}_{1} \backslash \bar{M}_{2}$ перейдут в 0 .

Рассмотрим элементы из $\bar{M}_{2}$. Тогда для всякого $h^{(t)} \in \bar{M}_{2}$ либо $D^{(t)} \neq D^{(1)}$ и, значит, элемент $h^{(t)}$ также перейдет в 0 , либо $D^{(t)}=D^{(1)}$ и тогда вместо $h^{(t)}$ появится произведение $q^{(t)}=q_{2}^{(t)} q_{3}^{(t)}$, где

$$
q_{3}^{(t)}=h^{(t)}\left[x_{q_{1}+1}, x_{q_{1}+2}\right] \cdots\left[x_{q_{1}+2 r-1}, x_{q_{1}+2 r}\right]
$$

а произведение $q_{2}^{(t)}$ получено из $h_{2}^{(t)}$ отбрасьванием множителя $x_{j_{1}}^{2} \cdots x_{j_{r}}^{2}$. Тогда

$$
q^{(t)}=q^{(t)}\left(x_{1}, \ldots, x_{q_{2}}\right)+V_{2}, \quad \text { где } q_{2}=q_{1}+2 r
$$

Таким образом, перенумеровывая еще раз, если нужно, слагаемые $\alpha_{t} g^{(t)}$ в элементе $f$, получаем элемент $f_{2 r}^{(2)}=\sum_{t=1}^{s^{\prime \prime}} \alpha_{t} q^{(t)}$, где для всех $t q^{(t)}$ - элементы вида (7), в которые ни один порождающий $x_{i}+V_{2}$ не входит одновременно в среднюю и конечную части, и количество порождаюших, входящих в среднюю часть, уменьшилось на $r$.

Положим

$$
\overline{\bar{M}}_{2}=\left\{q^{(t)} \mid t=1, \ldots, s^{\prime \prime}\right\}, \quad \overline{\bar{M}}_{3}=\left\{q^{(t)} \in \overline{\bar{M}}_{2} \mid h^{(t)} \in \bar{M}_{3}\right\} .
$$

Ясно, что $\overline{\bar{M}}_{3} \neq \varnothing$, так как $q^{(1)} \in \overline{\bar{M}}_{3}$.

Пусть

$$
q_{2}^{(1)}=x_{k_{1}}^{2} \cdots x_{k_{m}}^{2}+V_{2}, \quad m=m_{\max }-r .
$$

Положим

$$
\begin{aligned}
f_{0}^{(3)}=f_{2 r}^{(2)}, \quad f_{1}^{(3)} & =f_{0}^{(3)}+\psi_{k_{1}, q_{2}+1}\left(f_{0}^{(3)}\right)+\chi_{k_{1}, q_{2}+1}\left(f_{0}^{(3)}\right) \\
& =\sum_{t} \alpha_{t}\left(q^{(t)}+\psi_{k_{1}, q_{2}+1}\left(q^{(t)}\right)+\chi_{k_{1}, q_{2}+1}\left(q^{(t)}\right)\right) .
\end{aligned}
$$

Тогда

$$
\begin{aligned}
q^{(t)} & +\psi_{k_{1}, q_{2}+1}\left(q^{(t)}\right)+\chi_{k_{1}, q_{2}+1}\left(q^{(t)}\right) \\
& = \begin{cases}\tilde{q}_{2}^{(t)} q_{3}^{(t)}\left[x_{k_{1}}, x_{q_{2}+1}\right], & \text { если } x_{k_{1}}+V_{2} \text { входит в } q_{2}^{(t)} \\
0, & \text { если } x_{k_{1}}+V_{2} \text { входит в } q_{3}^{(t)}\end{cases}
\end{aligned}
$$


Положим далее

$$
\begin{aligned}
& f_{2}^{(3)}=f_{1}^{(3)}+\psi_{k_{2}, q_{2}+2}\left(f_{1}^{(3)}\right)+\chi_{k_{2}, q_{2}+2}\left(f_{1}^{(3)}\right) \\
& \ldots \ldots \ldots \ldots \ldots \ldots \ldots \ldots \ldots \ldots \ldots \ldots \ldots \ldots \ldots \ldots \ldots \ldots \ldots \ldots \ldots \ldots \ldots \ldots \ldots \ldots \ldots \\
& f_{m}^{(3)}=f_{m-1}^{(3)}+\psi_{k_{m}, q_{2}+m}\left(f_{m-1}^{(3)}\right)+\chi_{k_{m}, q_{2}+m}\left(f_{m-1}^{(3)}\right) .
\end{aligned}
$$

Рассмотрим элемент $f_{m}^{(3)} \in T$. Так как для всех элементов $q^{(t)} \in \overline{\bar{M}}_{2} \backslash \overline{\bar{M}}_{3}$ вьполняется $m_{t}<m$, то найдется порождающий $x_{k_{u}}+V_{2}$, которьй входит в $q_{2}^{(1)}$ и не входит в $q_{2}^{(t)}$ (т.е. входит в $\left.q_{3}^{(t)}\right)$, и, следовательно, все элементы из $\overline{\bar{M}}_{2} \backslash \overline{\bar{M}}_{3}$ перейдут в 0.

Рассмотрим элементы $q^{(t)} \in \overline{\bar{M}}_{3}$. Тогда либо $q_{2}^{(t)} \neq q_{2}^{(1)}$ и элемент $q^{(t)}$ также перейдет в 0 , либо $q_{2}^{(t)}=q_{2}^{(1)}$ и тогда вместо $q^{(t)}$ появится произведение

$$
\bar{q}^{(t)}=\bar{q}_{3}^{(t)}=q_{3}^{(t)}\left[x_{k_{1}}, x_{q_{2}+1}\right] \cdots\left[x_{k_{m}}, x_{q_{2}+m}\right] .
$$

В результате описанных выше преобразований элемента $f=\sum_{t} \alpha_{t} g^{(t)}$ не перешли в 0 только произведения $g^{(t)}$ вида (6) со следующими свойствами:

1) в $g^{(t)}$ входят в точности те же порождаюшие $x_{i}+V_{2}$, что и в $g^{(1)}$;

2) $g_{1}^{(t)}=g_{1}^{(1)}$

$3)$ в $\bar{g}^{(t)}$ входят в точности те же порождаюшие $x_{i}+V_{2}$, что и в $\bar{g}^{(1)}$ или, что эквивалентно, с учетом 2), в $g_{2}^{(t)} g_{3}^{(t)}$ входят в точности те же порождающие, что и в $g_{2}^{(1)} g_{3}^{(1)}$

4) $D^{(t)}=D^{(1)}$;

5) $q_{2}^{(t)}=q_{2}^{(1)}$, что эквивалентно, с учетом 4), равенству $g_{2}^{(t)}=g_{2}^{(1)}$.

Из свойств 3$)-5)$ следует, что в $g_{3}^{(t)}$ и в $g_{3}^{(1)}$ входят одни и те же порождающие $x_{i}+V_{2}$. Это означает, что $g_{3}^{(t)}=g_{3}^{(1)}$, откуда получаем $g^{(t)}=g^{(1)}$. Значит, единственный не перешедший в 0 в результате описанных вьше преобразований элемент $g^{(t)}$ - это элемент $g^{(1)}$.

Таким образом, получаем

$$
f_{m}^{(3)}=\alpha_{1}\left[x_{u_{1}}, x_{u_{2}}\right] \cdots\left[x_{u_{2 n-1}}, x_{u_{2 n}}\right]+V_{2} \in T
$$

для некоторых $n \in \mathbb{N}, \alpha_{1} \in F, \alpha_{1} \neq 0$ и некоторых попарно различных индексов $u_{1}, \ldots, u_{2 n}$. Откуда, применив подходящий автоморфизм алгебры $A / V_{2}$, действующий как подстановка на множестве порождающих, получаем

$$
\left[x_{1}, x_{2}\right]\left[x_{3}, x_{4}\right] \cdots\left[x_{2 n-1}, x_{2 n}\right]+V_{2} \in T
$$

что и требовалось доказать.

Пусть теперь $p>2$. Рассмотрим элементы множества $H$, т.е. произведения

$$
g=g_{1} g_{2}
$$

где

$$
\begin{aligned}
& g_{1}=x_{i_{1}}^{\beta_{1}} \cdots x_{i_{l}}^{\beta_{l}}+V_{p}, \quad i_{1}<\cdots<i_{l}, \quad 1 \leqslant \beta_{t}<p, \quad t=1, \ldots, l, \\
& g_{2}=\left[x_{j_{1}}, x_{j_{2}}\right] \cdots\left[x_{j_{2 m-1}}, x_{j_{2 m}}\right]+V_{p}, \quad j_{1}<\cdots<j_{2 m} .
\end{aligned}
$$


При этом одна из частей $g_{1}$ или $g_{2}$ может отсутствовать. Назовем $g_{1}$ начальной частью произведения $g, g_{2}$ - его конечной частью, а число $l$ - длиной произведения $g_{1}$.

Рассмотрим, как и в предыдущем случае, эндоморфизмы $\varphi=\varphi_{i, s, t}: A / V_{p} \rightarrow A / V_{p}$, полагая

$$
\varphi_{i, s, t}\left(x_{i}+V_{p}\right)=x_{i}+\left[x_{s}, x_{t}\right]+V_{p}, \quad \varphi_{i, s, t}\left(x_{j}+V_{p}\right)=x_{j}+V_{p} \text { для } j \neq i .
$$

Учитьвая, что $\left[x_{s}, x_{r}\right]^{2}+V_{p}=V_{p}$, нетрудно проверить, что если $g-$ произведение вида (8), то

$$
\varphi(g)= \begin{cases}g, & \text { если } x_{i}+V_{p} \text { не входит в } g_{1}, \\ g+\beta_{i} \tilde{g}_{1} g_{2}\left[x_{s}, x_{t}\right], & \text { если } x_{i}+V_{p} \text { входит в } g_{1} .\end{cases}
$$

Здесь $\tilde{g}_{1}=x_{i_{1}}^{\beta_{1}} \cdots x_{i}^{\beta_{i}-1} \cdots x_{i_{l}}^{\beta_{l}}+V_{p}$, т.е. $\tilde{g}_{1}$ - произведение, которое отличается от $g_{1}$ тем, что порождающий $x_{i}+V_{p}$ входит в $\tilde{g}_{1}$ в степени на 1 меньше. Получаем

$$
\varphi(g)-g= \begin{cases}0, & \text { если } x_{i}+V_{p} \text { не входит в } g_{1}, \\ \beta_{i} \tilde{g}_{1} g_{2}\left[x_{s}, x_{t}\right], & \text { если } x_{i}+V_{p} \text { входит в } g_{1} .\end{cases}
$$

Пусть теперь $f=\sum_{t=1}^{s} \alpha_{t} g^{(t)} \in T$, где для всех $t=1, \ldots, s \alpha_{t} \in F, \alpha_{t} \neq 0, g^{(t)}$ попарно различные произведения вида (8).

Пусть

$$
M_{0}=\left\{g^{(t)} \mid t=1, \ldots, s\right\} .
$$

Обозначим, как и раньше, символом $l_{t}$ длину начальной части $g_{1}^{(t)}$ произведения $g^{(t)}$. Пусть $\max _{t=1, \ldots, s} l_{t}=l_{\max }$. Положим

$$
M_{1}=\left\{g^{(t)} \in M_{0} \mid l_{t}=l_{\max }\right\}
$$

Пусть $g^{\left(t_{0}\right)}$ - некоторьй элемент из $M_{1}$,

$$
g_{1}^{\left(t_{0}\right)}=x_{i_{1}}^{\delta_{1}} \cdots x_{i_{l}}^{\delta_{l}}+V_{p}, \quad \text { где } \delta_{i}>0 \text { для } i=1, \ldots, l=l_{\max } .
$$

Положим

$$
M_{2}=\left\{g^{(t)} \in M_{1} \mid g_{1}^{(t)}=x_{i_{1}}^{\gamma_{1}} \cdots x_{i_{l}}^{\gamma_{l}}+V_{p}, \gamma_{1}, \ldots, \gamma_{l}>0\right\}
$$

Таким образом, $M_{2}$ - множество всех тех элементов $g^{(t)} \in M_{1}$, в начальную часть которых входят те же порождающие $x_{i_{1}}+V_{p}, \ldots, x_{i_{l}}+V_{p}$, что и в начальную часть $g^{\left(t_{0}\right)}$. Выберем $g^{(1)} \in M_{2}$ такой, что $g_{1}^{(1)}=x_{i_{1}}^{\beta_{1}} \cdots x_{i_{l}}^{\beta_{l}}+V_{p}$ и для любого произведения

$$
g^{(t)} \in M_{2}, \quad t \neq 1, \quad \text { где } g_{1}^{(t)}=x_{i_{1}}^{\gamma_{1}} \cdots x_{i_{l}}^{\gamma_{l}}+V_{p}
$$

либо $g_{1}^{(t)}=g_{1}^{(1)}$, либо найдется $r=r(t) \in \mathbb{N}$ такое, что $\beta_{r}>\gamma_{r}$, но $\beta_{u}=\gamma_{u}$ для всех $u<r$.

Пусть $q_{1}-$ максимальньй индекс такой, что $x_{q_{1}}+V_{p}$ входит в $f$. Положим

$$
f_{0}^{(1)}=f, \quad f_{1}^{(1)}=\varphi_{i_{1}, q_{1}+1, q_{1}+2}\left(f_{0}^{(1)}\right)-f_{0}^{(1)} .
$$


Тогда $f_{1}^{(1)} \in T$, причем $f_{1}^{(1)}=\sum_{t} \alpha_{t}\left(\varphi_{i_{1}, q_{1}+1, q_{1}+2}\left(g^{(t)}\right)-g^{(t)}\right)$ и $\varphi_{i_{1}, q_{1}+1, q_{1}+2}\left(g^{(t)}\right)-g^{(t)}= \begin{cases}0, & \text { если } x_{i_{1}}+V_{p} \text { не входит в } g_{1}^{(t)}, \\ \beta_{t 1} \tilde{g}_{1}^{(t)} g_{2}^{(t)}\left[x_{q_{1}+1}, x_{q_{1}+2}\right], & \text { если } x_{i_{1}}+V_{p} \text { входит в } g_{1}^{(t)} .\end{cases}$ (Здесь $\beta_{t 1}-$ степень, в которой $x_{i_{1}}$ входит в $g_{1}^{(t)}$.)

Положим далее

$$
\begin{aligned}
& f_{2}^{(1)}=\varphi_{i_{1}, q_{1}+3, q_{1}+4}\left(f_{1}^{(1)}\right)-f_{1}^{(1)} \\
& \ldots \ldots \ldots \ldots \ldots \ldots \ldots \ldots \ldots \ldots \ldots \ldots \ldots \ldots \ldots \ldots \ldots \ldots \ldots \ldots \\
& f_{\beta_{1}}^{(1)}=\varphi_{i_{1}, q_{1}+2 \beta_{1}-1, q_{1}+2 \beta_{1}}\left(f_{\beta_{1}-1}^{(1)}\right)-f_{\beta_{1}-1}^{(1)} .
\end{aligned}
$$

Посмотрим, что произойдет с каждьм из произведений $g^{(t)} \in M_{0}$ в результате такого процесса преобразования элемента $f=f_{0}^{(1)}=\sum_{t=1}^{s} \alpha_{t} g^{(t)}$ в $f_{\beta_{1}}^{(1)}$.

Пусть $g^{(t)} \in M_{0}$. Возможны следующие варианты. Если в произведение $g_{1}^{(t)}$ порождаюший $x_{i_{1}}+V_{p}$ не входит, то после первого шага $g^{(t)}$ перейдет в 0 . Если в произведение $g_{1}^{(t)}$ порождающий $x_{i_{1}}+V_{p}$ входит в степени $\gamma_{1}<\beta_{1}$, то на каждом шаге эта степень будет убьвать на 1 , и на каком-то из шагов произведение $g^{(t)}$ также перейдет в 0 . Если же в произведение $g_{1}^{(t)}$ порождаюший $x_{i_{1}}+V_{p}$ входит в степени $\beta_{1}$, то элемент $g^{(t)}$ перейдет в элемент $\bar{g}^{(t)}$, где $\bar{g}_{1}^{(t)}$ получается из $g_{1}^{(t)}$ исключением множителя $x_{i_{1}}^{\beta_{1}}$ (т.е. длина его начальной части уменьшилась на 1) и

$$
\bar{g}_{2}^{(t)}=g_{2}^{(t)}\left[x_{q_{1}+1}, x_{q_{1}+2}\right] \cdots\left[x_{q_{1}+2 \beta_{1}-1}, x_{q_{1}+2 \beta_{1}}\right] .
$$

В частности, $\bar{g}_{1}^{(1)}=x_{i_{2}}^{\beta_{2}} \cdots x_{i_{l}}^{\beta_{l}}+V_{p}$. Положим $q_{2}=q_{1}+2 \beta_{1}$.

Рассмотрим теперь элемент $f_{0}^{(2)}=f_{\beta_{1}}^{(1)}$. Ясно, что $f_{0}^{(2)} \in T$. Ясно также, что можно считать (перенумеровьвая, если нужно, слагаемые $\alpha_{t} g^{(t)}$ в элементе $f$ ), что $f_{0}^{(2)}=\sum_{t=1}^{s^{\prime}} \alpha_{t}^{\prime} \bar{g}^{(t)}$, где произведение $\bar{g}^{(t)}$ получено из $g^{(t)}$ описанными выше преобразованиями, а $\alpha_{t}^{\prime}=\alpha_{t} \beta_{1} \neq 0$ для всех $t$.

Положим

$$
\begin{aligned}
& f_{1}^{(2)}=\varphi_{i_{2}, q_{2}+1, q_{2}+2}\left(f_{0}^{(2)}\right)-f_{0}^{(2)}, \\
& f_{\beta_{2}}^{(2)}=\varphi_{i_{2}, q_{2}+2 \beta_{2}-1, q_{2}+2 \beta_{2}}\left(f_{\beta_{2}-1}^{(2)}\right)-f_{\beta_{2}-1}^{(2)} \text {, } \\
& f_{0}^{(3)}=f_{\beta_{2}}^{(2)} \text {, } \\
& f_{1}^{(3)}=\varphi_{i_{3}, q_{3}+1, q_{3}+2}\left(f_{0}^{(3)}\right)-f_{0}^{(3)}, \\
& f_{\beta_{l}}^{(l)}=\varphi_{i_{l}, q_{l}+2 \beta_{l}-1, q_{l}+2 \beta_{l}}\left(f_{\beta_{l}-1}^{(l)}\right)-f_{\beta_{l}-1}^{(l)},
\end{aligned}
$$

где $q_{u}=q_{u-1}+2 \beta_{u-1}$ для $u=2, \ldots, l$. Посмотрим, что произойдет с каждым произведением $g^{(t)} \in M_{0}$ в результате такого процесса преобразования элемента $f=f_{0}^{(1)}$ в $f_{\beta_{l}}^{(l)}$. 
Если элемент $g^{(t)} \in M_{0} \backslash M_{1}$, то $l_{t}<l$. Следовательно, найдется порождающий $x_{i_{j}}+V_{p}$, которьй входит в $g_{1}^{(1)}$, но не входит в $g_{1}^{(t)}$, и, значит, элемент $g^{(t)}$ перейдет в 0 .

Если элемент $g^{(t)}$ лежит в $M_{1} \backslash M_{2}$, то так как набор порождающих, входящих в $g_{1}^{(t)}$, не совпадает с набором порождающих, входящих в $g_{1}^{(1)}$, такое произведение $g^{(t)}$ также перейдет в 0 .

Если $g^{(t)} \in M_{2}$, то либо для некоторого порождающего $x_{i_{j}}+V_{p} \gamma_{j}<\beta_{j}$ и $\gamma_{u}=\beta_{u}$ для $u<j\left(\gamma_{u}-\right.$ степени, в которых $x_{i_{j}}+V_{p}$ входят в $\left.g_{1}^{(t)}\right)$, и тогда произведение $g^{(t)}$ перейдет в 0 уже в элементе $f_{\beta_{j}}^{(j)}$, либо $g_{1}^{(t)}=g_{1}^{(1)}$, тогда $g^{(t)}$ перейдет в итоге в элемент

$$
\overline{\bar{g}}^{(t)}=\overline{\bar{g}}_{2}^{(t)}=g_{2}^{(t)}\left[x_{q_{1}+1}, x_{q_{1}+2}\right] \cdots\left[x_{q_{l}+2 \beta_{l}-1}, x_{q_{l}+2 \beta_{l}}\right]
$$

т.е. в элемент вида (8), у которого отсутствует начальная часть.

Таким образом, перенумеровьвая еще раз, если необходимо, слагаемые $\alpha_{t} g^{(t)}$ в элементе $f$, получаем, что $f_{\beta_{l}}^{(l)}=\sum_{t=1}^{s^{\prime \prime}} \alpha_{t}^{\prime \prime} \overline{\bar{g}}^{(t)}$, где для всех $t$ имеем $\alpha_{t}^{\prime \prime} \in F, \alpha_{t}^{\prime \prime} \neq 0$, $\overline{\bar{g}}^{(t)}=g_{2}^{(t)} c, \mathrm{a}$

$$
c=\left[x_{q_{1}+1}, x_{q_{1}+2}\right] \cdots\left[x_{q_{l}+2 \beta_{l}-1}, x_{q_{l}+2 \beta_{l}}\right] .
$$

Как было показано вьше, для всех $t=1, \ldots, s^{\prime \prime}$ вьполнено $g_{1}^{(t)}=g_{1}^{(1)}$. Поскольку все произведения $g^{(t)}=g_{1}^{(t)} g_{2}^{(t)}$ попарно различны, а начальные части у них совпадают (и равны $g_{1}^{(1)}$ ), получаем, что конечные части $g_{2}^{(t)}$ этих произведений попарно различны. С учетом определения конечной части и тождества (4) это означает, что множества порождающих, входящих в $g_{2}^{(t)}$, попарно различаются.

Пусть теперь $\overline{\bar{g}}^{\left(t_{0}\right)}$ - произведение, в которое входит минимальное количество порождающих среди произведений, входящих в $f_{\beta_{l}}^{(l)}$, и пусть $x_{k_{1}}+V_{p}, \ldots, x_{k_{2 n}}+V_{p}$ - эти порождающие. Рассмотрим эндоморфизм $\tau=\tau_{k_{1}, \ldots, k_{2 n}}: A / V_{p} \rightarrow A / V_{p}$, где

$$
\tau\left(x_{k_{t}}+V_{p}\right)=x_{k_{t}}+V_{p} \text { для } t=1, \ldots, 2 n, \quad \tau\left(x_{j}+V_{p}\right)=0 \text { для } j \neq k_{1}, \ldots, k_{2 n} .
$$

Тогда $\tau\left(\overline{\bar{g}}^{\left(t_{0}\right)}\right)=\overline{\bar{g}}^{\left(t_{0}\right)}$ и $\tau\left(\overline{\bar{g}}^{(t)}\right)=0$ для всех $t \neq 0$, поэтому

$$
\tau\left(f_{\beta_{l}}^{(l)}\right)=\beta\left[x_{k_{1}}, x_{k_{2}}\right] \cdots\left[x_{k_{2 n-1}}, x_{k_{2 n}}\right]+V_{p} \in T
$$

где $\beta \in F, \beta \neq 0$.

Ясно, что $\tau\left(f_{\beta_{l}}^{(l)}\right) \in T$. Применим далее автоморфизм алгебры $A / V_{p}$, действуюший как подстановка на множестве порождающих, и получим

$$
\left[x_{1}, x_{2}\right]\left[x_{3}, x_{4}\right] \cdots\left[x_{2 n-1}, x_{2 n}\right]+V_{p} \in T
$$

что и требовалось доказать.

Теорема 3 доказана. 
3. Доказательство теоремы 5. Пусть $K$-нётерово коммутативно-ассоциативное кольцо с $1, A$ - свободная ассоциативная $K$-алгебра со свободными порождающими $x_{i}$, $i \in \Omega$. Нетрудно проверить, что достаточно доказать теорему в случае, когда $A-$ свободная алгебра без единицы счетного ранга, т.е. когда $\Omega=\mathbb{N}$.

Пусть $n$ и $N$ - произвольные натуральные числа, $\mathbf{V}=\mathbf{V}(n)$ - многообразие ассоциативных алгебр над $K$, задаваемое тождеством (1). Пусть $V-T$-идеал в $A$, соответствующий многообразию $\mathbf{V}$, так что $A / V$ - свободная алгебра многообразия $\mathbf{V}$ со свободными порождающими $x_{i}+V, i \in \mathbb{N}$. Пусть $S$ - множество всех элементов алгебры $A$, в которые каждый свободньй порождающий $x_{i}, i \in \mathbb{N}$, входит в степени не вьше $N$. Ясно, что $S-K$-подмодуль алгебры $A$. Для доказательства теоремы 5 нужно показать, что в $A / V$ конечно порождены все $T$-пространства, порожденные элементами из образа $\bar{S}=(S+V) / V$ множества $S$.

Обозначим символом $\Phi$ множество всех сохраняющих порядок отображений $\varphi$ множества натуральных чисел $\mathbb{N}$ в себя (т.е. таких $\varphi$, что если $k, l \in \mathbb{N}$ и $k<l$, то $\varphi(k)<\varphi(l))$. Тем же символом $\Phi$ мы будем обозначать множество всех таких эндоморфизмов $\varphi: A \rightarrow A$, что $\varphi\left(x_{i}\right)=x_{\varphi(i)}, i \in \mathbb{N}$. Обозначим символом $\Theta$ множество всех таких эндоморфизмов $\theta_{k l}: A \rightarrow A, k, l \in \mathbb{N}, k<l$, что

$$
\theta_{k l}\left(x_{i}\right)= \begin{cases}x_{k} x_{l}, & \text { если } i=l, \\ x_{i}, & \text { если } i \neq l .\end{cases}
$$

И, наконец, символом $\Lambda$ мы будем обозначать множество эндоморфизмов $\lambda: A \rightarrow A$ таких, что

$$
\lambda=\theta_{k_{1} l_{1}} \theta_{k_{2} l_{2}} \cdots \theta_{k_{q} l_{q}} \cdot \varphi,
$$

где $q \geqslant 0, \theta_{k_{1} l_{1}}, \theta_{k_{2} l_{2}}, \ldots, \theta_{k_{q} l_{q}} \in \Theta, \varphi \in \Phi, k_{i} \notin \varphi(\mathbb{N})$ при $i=1,2, \ldots, q, k_{i} \neq k_{j}$ при $i \neq j$.

Назовем $(\Lambda, K)$-подмодулем в $A K$-подмодуль, замкнутый относительно всех эндоморфизмов семейства $\Lambda$. Нетрудно проверить, что $S$ является $(\Lambda, K)$-подмодулем.

Пусть $I$ - идеал алгебры $A$, порожденный всеми коммутаторами $[f, g], f, g \in A$. Нетрудно проверить, что $I^{n}=V$, так что $A / V=A / I^{n}$. Так как $I$ является $(\Lambda, K)$-подмодулем и это же верно для $I^{k}$ при любом $k$, то понятие $(\Lambda, K)$-подмодуля естественным образом определено для факторалгебры $A / I^{k}$. Ясно, что $\bar{S}-(\Lambda, K)$-подмодуль в $A / V$.

Мы получим утверждение теоремы как следствие следующей леммы.

Лемма 1. Всякий $(\Lambda, K)$-подмодуль в $\bar{S}=\left(S+I^{n}\right) / I^{n}$ конечно порожден (как $(\Lambda, K)-$ подмодуль).

ДокАЗАТЕЛЬСТво леммы 1 будем проводить индукцией по $n$. При $n=0$ доказываемое утверждение верно, поскольку в этом случае $I^{n}=A$ и $A / I^{n}=\{0\}$.

Пусть теперь $n>0$, и пусть уже доказано, что в $\left(S+I^{n-1}\right) / I^{n-1}$ все $(\Lambda, K)$-подмодули конечно порождены. Пусть $U-$ произвольньй $(\Lambda, K)$-подмодуль в $S$. Пусть

$$
\bar{U}=\left(U+I^{n}\right) / I^{n}, \quad J=I^{n-1} / I^{n} .
$$

Чтобы доказать лемму 1 , нужно показать, что $\bar{U}$ - конечно порожденный $(\Lambda, K)$-подмодуль в $\bar{S}$. Ясно, что $\left(U+I^{n-1}\right) / I^{n-1}$ и $\bar{U} \cap J-(\Lambda, K)$-подмодули в $\left(S+I^{n-1}\right) / I^{n-1}$ и $\bar{S} \cap J$ соответственно. Если эти $(\Lambda, K)$-подмодули конечно порождены, то $\bar{U}-$ также 
конечно порожденный $(\Lambda, K)$-подмодуль, поскольку он является расширением $\bar{U} \cap J$ с помощью $\left(U+I^{n-1}\right) / I^{n-1} \simeq(\bar{U}+J) / J$. Заметим, что $\left(U+I^{n-1}\right) / I^{n-1}-$ конечно порожденный $(\Lambda, K)$-подмодуль по предположению индукции. Значит, лемма 1 будет доказана, если показать, что $\bar{U} \cap J$ - конечно порожденньй $(\Lambda, K)$-подмодуль. Конечная порожденность последнего $(\Lambda, K)$-подмодуля вытекает из следующей леммы.

Лемма 2. Всякий $(\Lambda, K)$-подмодуль в $\bar{S} \cap J$ конечно порожден.

ДоказАтЕльство. Достаточно показать, что в $\bar{S} \cap J$ стабилизируются любые возрастающие цепочки $(\Lambda, K)$-подмодулей.

Любой элемент из $J=I^{n-1} / V$ можно представить как линейную комбинацию элементов вида

$$
g_{1}\left[x_{m_{1}}, x_{m_{2}}\right] g_{2}\left[x_{m_{3}}, x_{m_{4}}\right] g_{3} \cdots\left[x_{m_{2 n-3}}, x_{m_{2 n-2}}\right] g_{n}+V,
$$

где для любого $i$ выполнено или $g_{i}=1$, или $g_{i}$ - (некоммутативньй) одночлен от порождаюших $x_{k}, k \in \mathbb{N}$, алгебры $A$. Заметим, что если $g_{i} \equiv g_{i}^{\prime}(\bmod I)$, то

$$
\begin{aligned}
& g_{1}\left[x_{m_{1}}, x_{m_{2}}\right] g_{2}\left[x_{m_{3}}, x_{m_{4}}\right] g_{3} \cdots\left[x_{m_{2 n-3}}, x_{m_{2 n-2}}\right] g_{n}+V \\
& \quad=g_{1}^{\prime}\left[x_{m_{1}}, x_{m_{2}}\right] g_{2}^{\prime}\left[x_{m_{3}}, x_{m_{4}}\right] g_{3}^{\prime} \cdots\left[x_{m_{2 n-3}}, x_{m_{2 n-2}}\right] g_{n}^{\prime}+V
\end{aligned}
$$

т.е. элементы $g_{i}$ в выражении (10) можно считать коммутативными одночленами от переменных $x_{k}$.

Пусть $M$ - множество всех (формальных) произведений вида (10) таких, что $m_{1}, m_{2}$, $\ldots, m_{2 n-2} \in \mathbb{N}$ и для любого $i$ множитель $g_{i}$ равен 1 или $g_{i}=x_{1}^{\alpha_{i 1}} x_{2}^{\alpha_{i 2}} \cdots x_{s_{i}}^{\alpha_{i s_{i}}}, \alpha_{i t} \geqslant 0$, $\alpha_{i s_{i}} \neq 0, i=1, \ldots, n$,

$$
\alpha_{1 t}+\alpha_{2 t}+\cdots+\alpha_{n t}+\tilde{d}_{t} \leqslant N
$$

для любого $t \in \mathbb{N}$, где $\tilde{d}_{t}-$ количество раз, которое число $t$ входит в последовательность $\left(m_{1}, m_{2}, \ldots, m_{2 n-2}\right)$. При $n=1$ определим $M$ как множество одночленов $g=$ $x_{1}^{\alpha_{1}} x_{2}^{\alpha_{2}} \cdots x_{s}^{\alpha_{s}}+I$ таких, что $\alpha_{s}>0,0 \leqslant \alpha_{t} \leqslant N$ для всех $t$. Нетрудно проверить, что любой элемент из $\bar{S} \cap J=\left(\left(S \cap I^{n-1}\right)+V\right) / V$ можно представить как линейную комбинацию элементов из $M$.

Подчеркнем, что мы рассматриваем элементы множества $M$ как формальные выражения: два различных выражения вида (10) считаются разными элементами множества $M$, даже если они представляют один и тот же элемент алгебры $A / V$ (например, если они оба равны 0). Введем на $M$ линейный порядок $\leqslant$. Пусть $a, a^{\prime} \in M$,

$$
\begin{aligned}
a & =x_{1}^{\alpha_{11}} x_{2}^{\alpha_{12}} \cdots\left[x_{m_{1}}, x_{m_{2}}\right] x_{1}^{\alpha_{21}} x_{2}^{\alpha_{22}} \cdots\left[x_{m_{2 n-3}}, x_{m_{2 n-2}}\right] x_{1}^{\alpha_{n 1}} x_{2}^{\alpha_{n 2}} \cdots, \\
a^{\prime} & =x_{1}^{\alpha_{11}^{\prime}} x_{2}^{\alpha_{12}^{\prime}} \cdots\left[x_{m_{1}^{\prime}}, x_{m_{2}^{\prime}}\right] x_{1}^{\alpha_{21}^{\prime}} x_{2}^{\alpha_{22}^{\prime}} \cdots\left[x_{m_{2 n-3}^{\prime}}, x_{m_{2 n-2}^{\prime}}\right] x_{1}^{\alpha_{n 1}^{\prime}} x_{2}^{\alpha_{n 2}^{\prime}} \cdots
\end{aligned}
$$

Обозначим символом $\leqslant$ лексикографический порядок на множестве $\mathbb{N}^{2 n-2}$, и тем же символом - лексикографический “справа налево" порядок на множестве $\{0,1, \ldots, N\}$. Положим $a<a^{\prime}$, если

$$
\left(m_{1}, m_{2}, \ldots, m_{2 n-2}\right)<\left(m_{1}^{\prime}, m_{2}^{\prime}, \ldots, m_{2 n-2}^{\prime}\right)
$$

или $m_{i}=m_{i}^{\prime}$ для всех $i$ и существует $s \in \mathbb{N}$ такое, что

$$
\left(\alpha_{1 s}, \alpha_{2 s}, \ldots, \alpha_{n s}\right)<\left(\alpha_{1 s}^{\prime}, \alpha_{2 s}^{\prime}, \ldots, \alpha_{n s}^{\prime}\right),
$$


но $\alpha_{j t}=\alpha_{j t}^{\prime}$ для всех $t>s$ и всех $j$. Ясно, что $\leqslant-$ полньй линейный порядок на $M$.

Пусть $a, b \in M, a>b$. Пусть $a$ имеет вид (11), и пусть

$$
\lambda=\theta_{k_{1} l_{1}} \cdots \theta_{k_{q} l_{q}} \cdot \varphi \in \Lambda, \quad \text { где }\left\{l_{1}, \ldots, l_{q}\right\} \cap\left\{\varphi\left(m_{1}\right), \ldots, \varphi\left(m_{2 n-2}\right)\right\}=\varnothing .
$$

Тогда можно проверить, что

$$
\lambda(a) \in M, \quad \lambda(b)=\sum_{i=1}^{v} b_{i}, \quad \text { где } b_{i} \in M, \quad \lambda(a)>b_{i} \quad \text { для всех } i=1, \ldots, v .
$$

Пусть $W=\{0,1,2, \ldots, N\}^{n} \times\{0,1\}^{2 n-2}$. Обозначим символом $V(W, 0)$ множество всех бесконечных последовательностей $\left\{w_{i}\right\}$ элементов из $W$, в которых почти все элементы $w_{i}$ равны $0=(0, \ldots, 0)$. Определим отображение $\xi: M \rightarrow V(W, 0)$, полагая

$$
\begin{aligned}
& \xi\left(x_{1}^{\alpha_{11}} x_{2}^{\alpha_{12}} \cdots\left[x_{m_{1}}, x_{m_{2}}\right] x_{1}^{\alpha_{21}} x_{2}^{\alpha_{22}} \cdots\left[x_{m_{2 n-3}}, x_{m_{2 n-2}}\right] x_{1}^{\alpha_{n 1}} x_{2}^{\alpha_{n 2}} \ldots\right) \\
& \quad=\left\{\left(\alpha_{1 i}, \alpha_{2 i}, \ldots, \alpha_{n i}, \gamma_{1 i}, \ldots, \gamma_{(2 n-2) i}\right)\right\}
\end{aligned}
$$

где

$$
\gamma_{t i}= \begin{cases}1, & \text { если } i=m_{t}, \\ 0, & \text { если } i \neq m_{t} .\end{cases}
$$

Ясно, что $\xi$ - инъективное отображение.

Определим на множестве $V(W, 0)$ преобразования $\varphi \in \Phi$ и $\theta_{k l} \in \Theta, k, l \in \mathbb{N}, k<l$, полагая $\varphi\left(\left\{a_{i}\right\}\right)=\left\{b_{i}\right\}$ и $\theta_{k l}\left(\left\{a_{i}\right\}\right)=\left\{c_{i}\right\}$, где

$$
b_{i}=\left\{\begin{array}{ll}
a_{j}, & \text { если } i=\varphi(j), \\
0, & \text { если } i \notin \varphi(\mathbb{N}) ;
\end{array} \quad c_{i}=\left\{\begin{array}{l}
a_{l}, \text { если } i=k, \\
a_{i}, \text { если } i \neq k .
\end{array}\right.\right.
$$

Наконец, определим на $V(W, 0)$ отображения $\lambda \in \Lambda$ так же, как в $(9)$, с теми же ограничениями на индексы $k_{i}$.

Рассмотрим на $V(W, 0)$ бинарное отношение $\leqslant \Lambda$, полагая $\left\{a_{i}\right\} \leqslant \Lambda\left\{b_{i}\right\}$ тогда и только тогда, когда существует такое $\lambda \in \Lambda$, что $\lambda\left(\left\{a_{i}\right\}\right)=\left\{b_{i}\right\}$. Из [17, лемма 3.3] следует, что $\leqslant \Lambda$ - полньй частичный порядок на $V(W, 0)$, т.е. что любая бесконечная последовательность $v_{1}, v_{2}, \ldots$ элементов из $V(W, 0)$ содержит бесконечную неубывающую подпоследовательность $v_{i_{1}} \leqslant \Lambda v_{i_{2}} \leqslant \Lambda \cdots, i_{1}<i_{2}<\cdots$.

Можно проверить, что если $a, a^{\prime} \in M, \xi(a) \leqslant \Lambda \xi\left(a^{\prime}\right)$, то существует такой эндоморфизм $\lambda \in \Lambda$, что $\lambda(a)=a^{\prime}$, причем если $a$ имеет вид (11) и $\lambda=\theta_{k_{1} l_{1}} \cdots \theta_{k_{q}} l_{q} \cdot \varphi$, то

$$
\left\{l_{1}, \ldots, l_{q}\right\} \cap\left\{\varphi\left(m_{1}\right), \ldots, \varphi\left(m_{2 n-2}\right)\right\}=\varnothing .
$$

Отсюда, используя стандартные рассуждения от противного (смотри, например [18, теорема 5.2.7]), мы получаем, что в $\bar{S} \cap J$ стабилизируются возрастающие цепочки $(\Lambda, K)$-подмодулей или, другими словами, что в $\bar{S} \cap J$ все $(\Lambda, K)$-подмодули конечнопорождены. Лемма 2 , а с ней и лемма 1 , доказаны.

Теперь все готово для завершения доказательства теоремы 5 . Пусть $U-$ произвольное $T$-пространство алгебры $A$, порожденное (как $T$-пространство) некоторыми элементами из $S$. Пусть $\bar{U}=(U+V) / V$. Ясно, что тогда $\bar{U}$ порождается множеством $\bar{U} \cap \bar{S}$, а $\bar{U} \cap \bar{S}$ является $(\Lambda, K)$-подмодулем в $\bar{S}$. По лемме $1 \bar{U} \cap \bar{S}$ - конечно порожденный $(\Lambda, K)$-подмодуль. Пусть $f_{1}+V, \ldots, f_{k}+V$ - порождающие этого $(\Lambda, K)$-подмодуля, тогда они же порождают $\bar{U}$ как $T$-пространство. Таким образом, $T$-пространство $\bar{U}$ конечно порождено, что и требовалось доказать.

Теорема 5 доказана. 


\section{СПИСОК ЦИТИРОВАННОЙ ЛИТЕРАТУРЫ}

[1] Бахтурин Ю.А., Ольшанский А. Ю. Тождества // Современные проблемы математики. Фундаментальные направления. Т. 18. М.: ВИНИТИ, 1988. С. 117-240.

[2] Бокуть Л.А., Львов И.В., Харченко В. К. Некоммутативные кольца // Современные проблемы математики. Фундаментальные направления. Т. 18. М.: ВИНИТИ, 1988. C. $5-116$.

[3] Drensky V.S. Free Algebras and PI-Algebras. Graduate Course in Algebra. Singapore: Springer-Verlag Singapore, 2000.

[4] Rowen L. H. Ring Theory. Boston: Academic Press, 1991.

[5] Specht W. Gesetze in Ringen // Math. Z. 1950. V. 52. P. 557-589.

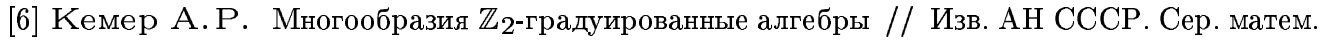
1984. T. 48. № 5. C. 1042-1059.

[7] Кемер А. Р. Конечная базируемость тождеств ассоциативных алгебр // Алгебра и логика. 1987. T. 26. C. $597-641$.

[8] Белов А.Я. О нешпехтовых многообразиях // Фундамент. и прикл. матем. 1999. Т. 5. C. $47-66$.

[9] Гришин А. В. Примеры не конечной базируемости $T$-пространств и $T$-идеалов в характеристике 2 // Фундамент. и прикл. матем. 1999. Т. 5. С. 101-118.

[10] Шиголев В. В. Примеры бесконечно базируемых $T$-идеалов // Фундамент. и прикл. матем. 1999. T. 5. C. $307-312$.

[11] Белов А. Я. Контрпримеры к проблеме Шпехта // Матем. сб. 2000. Т. 191. № 3. С. 13-24.

[12] Grishin A. V. On non-Spechtianness of the variety of associative rings that satisfy the identity $x^{32}=0 / /$ Electronic Research Announcements of the Amer. Math. Soc. 2000. №6. P. 50-51.

[13] Гришин А.В. О конечной базируемости систем обобщенных многочленов // Изв. АН СССР. Сер. матем. 1990. Т. 54. № 5. С. 899-927.

[14] Чирипов П. ЖК., Сидеров П. Н. О базисах тождеств некоторых многообразий ассоциативных алгебр // Плиска. 1981. Т. 2. С. 103-115.

[15] Шиголев В.В. Примеры бесконечно базируемых $T$-пространств // Матем. сб. 2000. T. 191. C. $143-160$.

[16] Латышев В.Н. О выборе базы в одном $T$-идеале // Сиб. матем. ж. 1963. Т. 4. №5. C. $1122-1126$.

[17] Красильников А. Н. О тождествах триангулируемых матричных представлений групп // Tp. MMO. 1989. T. 52. C. $229-245$.

[18] Бахтурин Ю. А. Тождества в алгебрах Ли. М.: Наука, 1985.

(Е.А. Киреева) Московский педагогический государственный университет

Поступило (А. Н. Красильников) Университет г. Бразилиа, Бразилия 15.10 .2004

E-mail : eakir@rol.ru, alexei@mat.unb.br 